\title{
Apolipoprotein E Polymorphism and Plasma Cholesterol Response to Probucol
}

\author{
A. Christine Nestruck, Daniel Bouthillier, Charles F. Sing, and Jean Davignon
}

Probucol has been shown to be an effective and well-tolerated cholesterol-lowering drug. However, response in terms of cholesterol reduction has been shown to vary significantly among individuals. The purpose of this study was to assess the role of apolipoprotein E polymorphism in determining this variation. A retrospective study of 89 hypercholesterolemic type II patients who had been treated with probucol $(1 \mathrm{~g} / \mathrm{d})$ and for whom the apolipoprotein $E$ phenotype was known was carried out. The patients were first grouped into those with heterozygous familial hypercholesterolemia (FH) and those considered to have other forms of hypercholesterolemia (non-FH). Further subclassification of the individuals in both groups as lla or Ilb, allowed the definition of four diagnostic classes, FH lla or Ilb and non-FH lla or llb. Among these classes there was no significant heterogeneity for the relationship between response and age or sex. After correction for between-class heterogeneity in duration of probucol treatment, comparison of individuals with the apo E3/3 phenotype with those carrying the 44 allele showed significant differences in cholesterol reduction both absolute change and percent change. Further contrasts between diagnostic and apo E genotype stratifications of these data showed that the FH patients carrying the 4 allele had the greatest reduction in cholesterol level.

1987 by Grune \& Stratton, Inc.

$\mathbf{A}^{1}$ LTHOUGH the efficacy of many lipid-lowering drugs is well-documented, it is also recognized that within the same diagnostic class of hyperlipidemia, with both dietary and drug therapies, there may be significant interpatient variability in response. There are generally no obvious characteristics of the patient that predict whether the individual will be a "responder" or "nonresponder," the degree of response, nor paradoxical increases in low-density lipoprotein cholesterol (LDL-C) which, for example, may occur in some patients nonresponsive to clofibrate. ${ }^{1-3}$ It is presumed that some of this variation in response is due to a genetic predisposition for differential metabolic effects of the lipidlowering intervention. This hypothesis is supported by the observation that approximately $50 \%$ of the interindividual variability in total serum cholesterol levels may be attributable to genetic influences. ${ }^{4}$ The human apolipoprotein $E$ (apo E) structural gene is polymorphic with three common alleles, $\epsilon 2, \epsilon 3$, and $\epsilon 4$, coding for three major isoforms. ${ }^{5-7}$ Evidence has accrued that apo $\mathrm{E}$ polymorphism influences the levels of plasma lipids and lipoproteins, ${ }^{8.11}$ a striking example being that the homozygous E2/2 phenotype (and occasionally E2 heterozygosity) is necessary, in combination with other genetic or nongenetic influences, for the development of familial dysbetalipoproteinemia (type III).$^{12-14}$ By extension, several studies have searched for an influence of the apo $E$ gene on the development of atherosclerosis. ${ }^{15-17}$ Additionally, from a number of studies of normal individuals apo $E$ has been shown to account for $9 \%$ to $15 \%$ of the normal variability in plasma cholesterol levels; the $\epsilon 2$ allele predicting a lower and the $\epsilon 4$ allele a higher plasma cholesterol concentration. ${ }^{18,19}$

Probucol has been shown to have a variable efficacy in the treatment of hypercholesterolemia type II; the genetic classification (monogenic or polygenic) partially predicting the degree of LDL-C reduction. ${ }^{20}$ Preliminary evidence had suggested to us that patients with the $\epsilon 4$ allele responded better, in terms of plasma cholesterol reduction, to probucol. ${ }^{21}$ We therefore undertook this retrospective, systematic study, using a much larger number of patients, to determine whether the lipid-lowering effect of probucol is independent of the apolipoprotein $E$ genotype of the patient.

\section{MATERIALS AND METHODS}

\section{Subjects}

The clinical records of 131 hypercholesterolemic type II patients of our lipid clinic who had been given probucol were surveyed. Having satisfied the following criteria, 89 were entered into this study: the patients had high plasma cholesterol levels and LDL-C $>190 \mathrm{mg} / \mathrm{dL}$, probucol $(1 \mathrm{~g} / \mathrm{d})$ was the only lipid-lowering drug administered and there were no cases with confounding secondary factors such as oral synthetic hormone replacement (estrogens or thyroxin), ileal bypass, or diabetes. A stable baseline period with the patients taking no lipid-lowering drugs but on a prescribed diet, appropriate to the hyperlipidemia phenotype, was chosen to include the three or four cholesterol measurements that immediately preceded the probucol period. In ten cases, however, because other therapeutic regimens had been tried in the interval, this was not available and the baseline had been obtained $2.7 \pm 1.0$ years previously. In five of these cases, however, one or two cholesterol values were available prior to the administration of probucol which were very similar to the baseline obtained earlier, or with cessation of probucol, cholesterol levels rose to the range of the previously obtained baseline. There was no clustering of these ten subjects in any of the diagnostic or genotype subgroups: $5 \mathrm{FH}, 5$ non-FH with almost equal distribution of IIa and IIb classifications. Seven subjects had the E3/3 phenotype and the three apo E4/3 individuals were in the group of subjects for whom at least one cholesterol observation was available just prior to institution of probucol trcatment. Further, cholesterol response to probucol was not extreme in these ten subjects; all showed moderate reductions. The baseline averaged $14 \pm 6$ weeks. In order to have enough determinations to assess the effect of probucol, the period of observation was selected as the first five or six cholesterol measurements after institution of the drug and this averaged $28 \pm 12$ weeks. Dietary therapy

From the Department of Lipid Metabolism and Atherosclerosis Research, Clinical Research Institute of Montreal, and the Department of Human Genetics, University of Michigan.

Supported in part by grants from the Medical Research Council of Canada, (MA-8757, MT-5427), the Quebec Heart Foundation, and the NIH (HL24489, CA26803).

Address reprint requests to A.C. Nestruck, PhD, 110 Pine Ave West, Montreal, $P$ Quebec, Canada H2W IR7.

(c) 1987 by Grune \& Stratton, Inc.

0026-0495/87/3608-0007\$03.00/0 
Table 1. Clinical Features and Details of the Study Periods

\begin{tabular}{|c|c|c|c|c|}
\hline \multirow{2}{*}{$\frac{\text { Hyperlipidemia }}{\text { Total }}$} & \multirow{2}{*}{$\frac{n \text { (Males) }}{89(36)}$} & \multirow{2}{*}{$\frac{\text { Apo E Phenotype (n) }}{\text { E4/4 (3) }}$} & \multicolumn{2}{|c|}{ Study Periods } \\
\hline & & & Weeks on baseline & $13.9 \pm 6.4$ \\
\hline & & $3 / 3(57)$ & Baseline measurements, $n$ & $3.7 \pm 1.2$ \\
\hline FH lla & $34(11)$ & $2 / 2(1)$ & Weeks on probucal & $27.7 \pm 11.7$ \\
\hline FH IIb & $16(9)$ & $4 / 3(20)$ & Probucol measurements, $n$ & $5.1 \pm 2.0$ \\
\hline Ila & $12(1)$ & $3 / 2(6)$ & & \\
\hline IIb & $27(15)$ & $4 / 2(2)$ & & \\
\hline
\end{tabular}

Values are given as mean $\pm \mathrm{SD}$.

continued during the probucol period and was monitored throughout by regular consultations with a dietitian and as a routine, by body weight fluctuation.

The diagnosis of familial hypercholesterolemia (FH) was confirmed on the basis of raised cholesterol levels associated with the presence of tendon xanthomas $(X)$, which in doubtful cases had been confirmed by radiologic examination, or a positive family history for premature coronary heart disease and tendon xanthomas in at least one first-degree relative. Although many of the type II patients had been classified as familial combined hyperlipidemia $(\mathrm{FCH})$ due to the presence of multiple hypcrlipidemic phenotypes within the same family, this information was not available for all patients, and therefore, this non-FH group included $\mathrm{FCH}$ and possibly polygenic hypercholesterolemia. ${ }^{22}$ Both FH and non-FH type II were subclassified as IIa or Ilb on the basis of baseline plasma triglyceride:cholesterol ratios of $<0.4$ and $\geq 0.4$, respectively. ${ }^{1}$ This resulted in four diagnostic classes: IlaX and IIbX for FH and IIa and IIb for non-FH. The clinical features of the patients and the study periods are summarized in Table 1.

All blood samples were obtained after 12 to 14 hours of fasting and drawn into Vacutainer tubes containing EDTA $(1 \mathrm{mg} / \mathrm{mL}$. Plasma were stored at $4^{\circ} \mathrm{C}$ until analysis within three to four days. As previously described, plasma and lipoprotein lipids were measured using an autoanalyser (ABA-100, Abbott Laboratories) after Lipid Research Clinic methods and apolipoprotein $\mathrm{E}$ phenotypes were determined after isoelectric focusing of VLDL separated and washed by ultracentrifugation. ${ }^{23}$

\section{Statistical Methods}

The average levels of cholesterol during the baseline period and the period while the patient was on probucol were the primary variables considered. Multiple linear regression ${ }^{24}$ was used to investigate the contribution of age at the beginning of the probucol treatment, number of weeks on baseline, weeks on probucol, number of times cholesterol was measured during the baseline and number of measurements during the probucol period to interindividual variation in these primary variables. The one-way ANOVA was used to test for significant differences in the average value of a trait among diagnostic classes and apoE genotypes. ${ }^{24}$ Statistical tests were judged significant at the .05 level of probability.

Table 2. Average Age and Weeks on Baseline for Each of the Four Subgroups of Patients

\begin{tabular}{cccc}
\hline & \multicolumn{3}{c}{ Four Subgroups of Patients } \\
\hline FH lla & $n$ & Age $(\mathrm{yr})$ & Weeks on Baseline \\
FH llb & 34 & $43.9 \pm 13.3$ & $13.1 \pm 6.0$ \\
Ila & 16 & $44.7 \pm 9.2$ & $10.1 \pm 5.1$ \\
Ilb & 12 & $55.0 \pm 17.2$ & $16.0 \pm 5.7$ \\
$F$ test & 27 & $50.9 \pm 9.8$ & $16.4 \pm 6.9$ \\
Probability & & 3.39 & 4.32 \\
\hline
\end{tabular}

Values are given as mean $\pm \mathrm{SD}$.

\section{RESULTS}

We began our analyses of these data by considering the distribution among the diagnostic classes and apo $E$ genotypes of age at the beginning of the probucol treatment, weeks on baseline, number of measurements during the baseline period, weeks on probucol, and the number of measurements during the probucol period. We found no statistically significant differences among genotypes for the average of any of these concomitants. However, the average age of the patients and weeks on baseline varied significantly among the four diagnostic classes. Table 2 summarizes these comparisons.

The comparison of the average age of patients with $\mathrm{FH}$ (44.2 years) with that of the non-FH patients (52.1 years) explains the major fraction of the variability among diagnostic classes. The non-FH individuals were followed significantly longer ( 4 more weeks) during the baseline period than those with FH. This difference in length of time, however, was not associated with a significant difference in the number of measurements during the baseline.

We next turned to determining whether these concomitants were associated with individual average cholesterol levels during baseline or the probucol periods. Twenty-one percent $(P<.001)$ of the interindividual variability in average cholesterol for the baseline period and $8 \%(P>.10)$ for the probucol period were associated with variability in these five concomitants. As summarized in Table 3, a stepwise regression established that weeks on baseline and weeks on probucol explain a major fraction of the variability in baseline cholesterol, $\mathrm{R}^{2}=17.6$. Although the effect of these predictors on the average cholesterol level during the probucol period was not statistically significant, the longer that the patient was followed the lower the average choles-

Table 3. Regression Coefficients for the Relationship Between the Dependent Variables, Mean Baseline (B) Cholesterol, and Mean Probucol (PB) Chalesterol, and Concomitant Variation in Age, Weeks of Treatment, and Number of Observations During Each Period

\begin{tabular}{lcc}
\hline \multicolumn{1}{c}{ Concomitant } & $\begin{array}{c}\text { Baseline } \\
\text { Cholesterol }\end{array}$ & $\begin{array}{c}\text { Probucol } \\
\text { Cholesterol }\end{array}$ \\
\hline Age & 0.22 & 0.20 \\
Weeks B & $-2.97 *$ & -2.06 \\
Samples B (n) & -14.37 & -6.25 \\
Weeks PB & $-2.45^{*}$ & -1.01 \\
Samples PB (n) & 5.68 & 1.05 \\
$\mathrm{R}^{2}$ & $21.03^{*}$ & 8.3 \\
\hline Significantly different from zero at the .01 level of probability.
\end{tabular}


Table 4. A Description of the Cholesterol Date

\begin{tabular}{lccc}
\hline \multicolumn{1}{c}{ Variable } & Mean & SD & Range of Values \\
\hline $\begin{array}{l}\text { Raw unadjusted data } \\
\text { Baseline }\end{array}$ & 363.47 & 74.75 & $229-598$ \\
Probucol & 313.01 & 65.81 & $195-520$ \\
Change & -50.46 & 41.65 & $71--144$ \\
\% Change & -13.39 & 11.00 & $24.5--39.0$ \\
Adjusted data & & & \\
Baseline & 363.47 & 67.85 & $208-577$ \\
Probucol & 313.01 & 63.38 & $185-489$ \\
Change & -50.46 & 39.20 & $61--138$ \\
\% Change & -13.52 & 10.34 & $20.2--38.3$ \\
\hline
\end{tabular}

The change variable $=$ the average level during the baseline - the average level during the probucol treatment period.

terol level in both periods. There was no evidence for significant heterogeneity (at .10 level of probability) among diagnostic classes or among the apoE genotype classes for either of the regression equations given in Table 3 .

We next adjusted the individual average cholesterol levels during the baseline and during the probucol periods each separately for weeks on baseline and weeks on probucol. Table 4 presents a description of the unadjusted and adjusted data that will be considered below for the analysis of the effects of diagnosis and apolipoprotein $\mathrm{E}$ genotype on response to probucol. After adjustment, the average values do not change but the range is narrowed and there are small reductions in the standard deviations of each of the four variables. A paired $t$ test of the average of the difference between individual mean cholesterol levels during baseline and probucol was significant at the .001 level of probability.

All subjects did not respond to probucol with a drop in cholesterol. Although 81 patients showed an average decrease of $58 \mathrm{mg} / \mathrm{dL}$ (adjusted data, $14.6 \%$ change), the mean plasma cholesterol increased in eight. These records were surveyed in detail. All patients showed increases in cholesterol and LDL-C and the baseline had been obtained immediately before the probucol period. Seven of these resistant patients have the E3/3 genotype (four FH type IIa, one IIa non-FH and two FH IIb). The other resistant individual is E3/2 and type IIa FH.

For comparison of the adjusted data by genotype and diagnostic classification, the one E2/2 and six E3/2 individuals were not considered because of small sample sizes. We include here only the contrast of the most frequent E3/3 genotypic class $(n=57)$ with the pooled class of genotypes carrying the $\epsilon 4$ allele $(\mathrm{E} 4 / 2+\mathrm{E} 4 / 3+\mathrm{E} 4 / 4, \mathrm{n}=25)$. There were highly significant differences among the diagnostic classes at both baseline and during the probucol treatment. For the baseline period, patients with FH (IIaX and IIbX) had a significantly higher $(P<.001)$ average cholesterol $(388 \pm 61 \mathrm{mg} / \mathrm{dL})$ than the non-FH group $(329 \pm 58$ $\mathrm{mg} / \mathrm{dL}$ ). The differences between the mean IIaX $v$ IIbX and IIa $v$ IIb, during the baseline period or during the probucol treatment, were not statistically significant. Despite the differences in baseline levels, the average response of the $\mathrm{FH}$ group $(-51 \pm 39 \mathrm{mg} / \mathrm{dL})$ was not significantly different from the average response of the non-FH group $(-49 \pm 37$ $\mathrm{mg} / \mathrm{dL}),-12.9$ and $-15.2 \%$, respectively.

\section{CHOLESTEROL $\mathrm{mg} / \mathrm{dl}$}

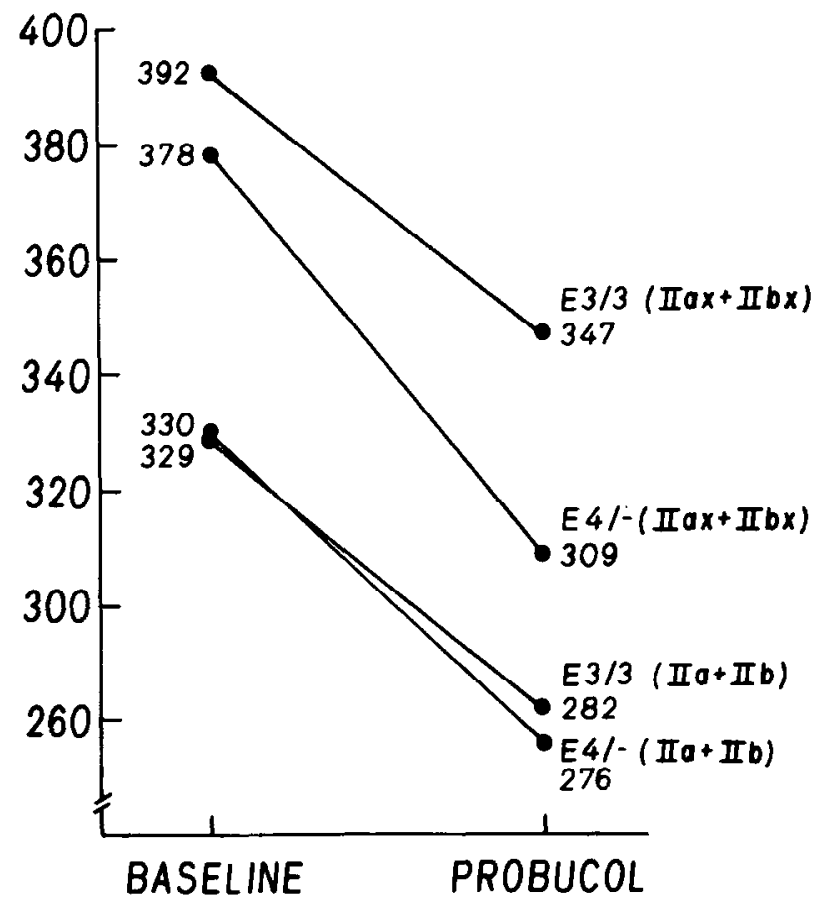

Fig 1. Mean plasma cholesterol levels for type II hypercholesterolemic patients on baseline (no lipid-lowering medication) and on probucol $(1 \mathrm{~g} / \mathrm{d})$. Subjects were subgrouped by diagnosis, Familial Hypercholesterolemia (FH) (Ilax + Ilbx) and non-FH (Ila + 11b). and by apo E genotype, E3/3 FH (n = 35) and non-FH in $=22$ ) and $E 4 /-,(E 4 / 4+4 / 3+4 / 2) F H(n=11)$ and non-FH $(n=14)$.

In considering response by genotype, however, although the average baseline cholesterol level of the $\mathrm{E} 4$ - genotypes was not significantly different than the average of the E3/3 genotypes, there was a highly significant difference between genotypes on probucol. This effect is reflected in a significantly larger response for the E4- $(-61 \pm 36 \mathrm{mg} / \mathrm{dL}$, $-18 \%)$ than the E3/3- genotypes $(-46 \pm 39 \mathrm{mg} / \mathrm{dL}$, $-12 \%$ ). Furthermore, as shown in Fig 1, this response is dependent on the diagnostic classification. For the patients with presumably polygenic hypercholesterolemia and familial combined hypercholesterolemia, ie non- $\mathrm{FH}$, the response to probucol was not significantly different between genotypes, the E3/3 and E4 - groups showing similar reductions, -47 and $-54 \mathrm{mg} / \mathrm{dL}$ change, $-14 \%$ and $-16 \%$ respectively, $P>.50$. In contrast, for those with $\mathrm{FH}$, the drop in cholesterol was significantly greater in those subjects with the E4 allele $(-70 \pm 39 \mathrm{mg} / \mathrm{dL},-18 \%)$ than in those with the E3/3 genotype $(-45 \pm 39 \mathrm{mg} / \mathrm{dL},-11 \%), P<.03$.

\section{DISCUSSION}

This retrospective study confirms and extends the observations of previous trials ${ }^{2,3,20,25-29}$ in which probucol was shown to be a moderately effective cholesterol lowering agent in diet-resistant hyperlipidemia type II with significant interpatient variability in response. In an attempt to investigate 
genetic factors which may play a role in this variability, we first subdivided the patients into two groups; those with clearly defined familial hypercholesterolemia and those with FCH or hypercholesterolemia of undefined polygenic etiology, or non-FH. The FH patients were found to be younger and were followed an average of 4 weeks less during the baseline period. Both groups were found to have significantly elevated cholesterol levels at baseline, and notwithstanding the higher baseline cholesterol levels of the $\mathrm{FH}$ group during baseline, both $\mathrm{FH}$ and non-FH groups responded to probucol with an average drop of approximately $50 \mathrm{mg} / \mathrm{dL}$, again with significant interpatient variability and with no differences between the IIa or IIb subclassifications. For the eight patients whose cholesterol and LDL-C levels showed an average rise with probucol, five had been diagnosed as $\mathrm{FH}$ IIa, two as FH IIb, and one as IIa non-FH.

When response was considered in terms of apo E genotype, however, the genotypes with the $\epsilon 4$ allele were found to have a significantly greater reduction in cholesterol level than the E3/3 genotype. Furthermore, when diagnostic classification was considered in addition to apo $\mathrm{E}$ genotype, this effect was shown to be due to the significantly greater response of the FH patients having the $\epsilon 4$ allele; $\mathrm{E} 3 / 3 \mathrm{FH}$, non-FH patients with the $\epsilon 4$ allele and the non-FH E3/3 patients all responding with smaller reductions in cholesterol level.

This study was not designed to investigate changes in cholesterol associated with the individual lipoprotein classes. However, in view of the lowering effect of probucol on both LDL and HDL cholesterol, ${ }^{26,27}$ we were interested in the contribution of potential reductions in IIDL-C to the observed overall changes in plasma cholesterol levels. We were able to obtain one or two lipoprotein cholesterol profiles in both the baseline and probucol periods for 30 of the 82 subjects whose cholesterol values were considered in the contrasts of genotype by diagnostic classification: in the $\mathrm{FH}$ group, $11 / 35 \mathrm{E} 3 / 3$ and $5 / 11 \mathrm{E} 4 /-$, and in the non-FH group, $8 / 22 \mathrm{E} 3 / 3$ and $6 / 14 \mathrm{E} 4 /-$ individuals. The initial HDL-C levels for all subjects were low and the changes observed were similar in all sub-groups: $-31+16 \%$ from $43 \pm 8 \mathrm{mg} / \mathrm{dL}$ for the FH E3/3 subjects, $-33 \pm 13 \%$ from $40 \pm 12 \mathrm{mg} / \mathrm{dL}$ for the FH E4/ - patients, $-33 \pm 23 \%$ from $42 \pm 9 \mathrm{mg} / \mathrm{dL}$ for the non-FH E3/3 subjects and $-27 \pm 14 \%$ from $41 \pm 8 \mathrm{mg} / \mathrm{dL}$ for the non-FH E4/ - patients. Thus, in this study, the response of total cholesterol to probucol and the differences attributed to genotypic and diagnostic classification appear to reflect changes in L.DI -C. .

In previous studies of response to probucol, attempts have been made to explain variability in response on the basis of diagnostic classification of the hyperlipidemia ${ }^{2,20,27,29}$ with only partial success. This study clearly demonstrates that the nature of the response to probucol depends on the genetic determination of the hypercholesterolemia as well as the apolipoprotein $\mathrm{E}$ genotype. The mechanisms underlying this association are at best speculative.

Current evidence, from the findings of Baker et $\mathrm{al}^{30}$ that homozygous, receptor-negative FH patients respond well to probucol with lowered LDL cholesterol levels and regression of xanthomas and the findings of Naruszewicz et $\mathrm{al}^{31}$ that receptor-deficient WHHL rabbits treated with probucol show substantial reductions in LDL cholesterol and an increased catabolic rate of LDL, suggests that one of the possible modes of action of probucol does not involve the LDL receptor, the genetic defect in FH. A receptormediated process may also, however, be superimposed on this mode of action. ${ }^{28}$ Again, the studies using fibroblasts from both WHHL and normal rabbits and in vivo injections of LDL from probucol treated animals, demonstrated an increased uptake and degradation of LDL from probucol treated animals by the high affinity LDL receptor possibly due to a chemically induced change in the LDL molecules. ${ }^{30}$ Probucol is a unique lipid-lowering agent due to its sulfurcontaining lipophilic properties, ${ }^{32}$ and it is a powerful antioxidant estimated to be more potent than vitamin $E .^{33}$ Further, it was recently reported that probucol added to in vitro incubations of LDL with endothelial cells is a highly effective inhibitor of cell-mediated oxidation of LDL and thus directs catabolism away from the macrophage pathway. ${ }^{34,35}$ The observed regression in xanthomas ${ }^{30}$ further supports the premise that this action is also independent of the acetylLDL-receptor of the macrophages. Thus, it is likely that probucol enhances the metabolism of LDL by mechanisms independent of the native or modified-LDL receptor.

Apolipoprotein $\mathrm{E}$ is a major determinant responsible for mediating the high affinity binding of lipoproteins to the $\mathrm{LDL}$ receptor as well as the hepatic apo $\mathrm{E}$ receptor. ${ }^{36}$ The major isoforms are known to differ in their ability to interact with these receptors; the E2 form and various E2 mutants having as little as $2 \%$ of the normal E3 binding ${ }^{36}$ which results in varying degrees of decreased remnant catabolism. The E4 isoform has equivalent binding to E3; the structural modification of an additional positively charged residue in E4 at site 112 having no apparent effect on binding. ${ }^{36}$ Recently, however, it has been reported that E4 VLDL is catabolized more rapidly in E3/3 individuals and independently of the LDL receptor. ${ }^{37}$ Thus, taken with the probucol induced chemical modifications of LDL which result in an altered and enhanced LDL catabolism, it is possible that an additionally enhanced catabolism of VLDL, both processes being independent of the LDL receptor, explains the greater response of FH- $\epsilon 4$ carrying individuals in the present study. A presumption being that in the face of defective receptors (FH), the role of apo $E$ possibly as a determinant of lipoprotein conformation ${ }^{38}$ is crucial in determining lipoprotein metabolism. That the non-FH individuals did not respond as well can be explained by the fact that the underlying genetic etiology of their disease involves many gene loci rather than a monogenic receptor defect. The polygenic nature of the non-FH hypercholesterolemia likely masks the apo $\mathrm{E}$ response that is observed in $\mathrm{FH}$ patients. The use of probucol as a probe provides further evidence for the role of genetic factors in determining the homenstasis that establishes individual serum cholesterol levels. Genetic factors must be considered in our efforts to understand the mechanism(s) of action of probucol and in our selection of intervention strategies to lower an individual's cholesterol lcvel. 


\section{REFERENCES}

1. Davignon J, Aubry, Noel C, et al: Heterogeneity of familial hyperlipoproteinemia type II on the basis of fasting plasma triglyceride/cholesterol ratio and plasma cholesterol response to ethyl p-chlorophenoxyisobutyrate. Rev Can Biol Exp 30:307-318, 1971

2. LeLorier J, DuBreuil-Quidoz S, Lussier-Cacan S, et al: Diet and probucol in lowering cholesterol concentrations. Arch Intern Med 137:1429-1434, 1977

3. Enjalbert M, Lussier-Cacan S, DuBreuil-Quidoz S, et al: Usefulness of probucol in treating primary hypercholesterolemia. Can Med Assoc J 123:754-757, 1980

4. Sing CF, Orr JD: Analysis of genetic and environmental sources of variation in serum cholesterol in Tecumseh, Michigan. IV. Separation of polygene from common environmental effects. Am J Hum Genet 30:491-504, 1978

5. Utermann G, Hees M, Steinmetz A: Polymorphism of apolipoprotein $E$ and occurrence of dysbetalipoproteinemia in man. Nature 269:604-607, 1977

6. Zannis VI, Breslow JL: Human very low density lipoprotein apolipoprotein $\mathrm{E}$ isoprotein polymorphism is explained by genetic variation and post-translational modification. Biochemistry 20:1033-1041, 1981

7. Zannis VI, Breslow JL, Utermann G, et al: Proposed nomenclature of apo $E$ isoproteins, apo $E$ genotypes and phenotypes. J Lipid Res 23:911-914, 1982

8. Ghiselli G, Schaefer EJ, Zech LA, et al: Increased prevalence of apolipoprotein $\mathrm{E} 4$ in type $\mathrm{V}$ hyperlipoproteinemia. J Clin Invest 70:474-477, 1982

9. Assmann G, Schmitz G, Menzel H-J, et al: Apolipoprotein E polymorphism and hyperlipidemia. Clin Chem 30:641-643, 1984

10. Davignon J, Sing CF, Lussier-Cacan S, et al: Xanthelasma, latent dyslipoproteinemia and atherosclerosis: contribution of apo $\mathrm{E}$ polymorphism, in DeGennes JL, Polonowsky J, Paoletti R (eds): Latent Dyslipoproteinemia and Atherosclerosis. New York, Raven, 1984, pp 213-223

11. Utermann G, Kindermann I, Kaffarnik H, et al: Apolipoprotein $E$ phenotypes and hyperlipidemia. Hum Genet 65:232-236, 1984

12. Havel RJ: Familial dysbetalipoproteinemia-new aspects of pathogenesis and diagnosis. Med Clin North Am 66:441-454, 1982

13. Breslow JL, Zannis VI, Sangiacomo TR, et al: Studies of familial type III hyperlipoproteinemia using as a genetic marker the apo E phenotype E2/2. J Lipid Res 23:1224-1235, 1982

14. Ghiselli G, Gregg RE, Zech LA, et al: Phenotype study of apolipoprotein $\mathrm{E}$ isoforms in hyperlipoproteinemic patients. Lancet 2:405-407, 1982

15. Menzel HJ, Kladetsky RG, Assmann G: Apolipoprotein E polymorphism and coronary artery disease. Arteriosclerosis 3:310322, 1983

16. Cumming AM, Robertson FW: Polymorphism at the apoprotein $\mathrm{E}$ locus in relation to risk of coronary disease. Clin Genet 25:310-313, 1984

17. Utermann G, Hardewig A, Zimmer F: Apolipoprotein E phenotypes in patients with myocardial infarction. Hum Genet 65:237-241, 1984

18. Sing CF, Davignon J: Role of apolipoprotein E polymorphism in determining normal plasma lipid and lipoprotein variation. Am J Hum Genet 37:268-285, 1985

19. Boerwinkle E, Visvikis S, Welsh D, et al: The use of measured genotype information in the analysis of quantitative phenotypes in man. II. The role of the apolipoprotein E polymorphism in deter- mining levels, variability and covariability of cholesterol, betalipoprotein and triglycerides in a sample of unrelated individuals. Am J Med Genet (in press)

20. Cortese C, Marenah CB, Miller NE, et al: The effects of probucol on plasma lipoproteins in polygenic and familial hypercholesterolaemia. Atherosclerosis 44:319-325, 1982

21. Bouthillier D: Effects du probucol sur les différentes lipoprotéines et apolipoprotéines humaines. Doctoral thesis, University of Montreal, 1982

22. Goldstein JL, Hazzard WR, Schrott HG, et al: Hyperlipidemia in coronary heart disease II. Genetic analysis of lipid levels in 176 families and delineation of a new inherited disorder, combined hyperlipidemia. J Clin Invest 52:1544-1568, 1973

23. Bouthillier D, Sing CF, Davignon J: Apolipoprotein E phenotyping with a single gel method: application to the study of informative matings. J L ipid Res 24:1060-1069, 1983

24. Neter J, Wasserman W, Kutner MH: Applied linear statistical models. Homewood, IL, RD Irwin, 1985

25. Hunninghake DB, Bell C, Olson L: Effect of probucol on plasma lipids and lipoproteins in type IIb hyperlipoproteinemia. Atherosclerosis $37: 469-474,1980$

26. Mellies MJ, Gartside L, Glatfelder P, et al: Effects of probucol on plasma cholesterol, high and low density lipoprotein cholesterol, and apolipoproteins A1 and A2 in adults with primary familial hypercholesterolemia. Metabolism 29:956-964, 1980

27. Atmeh RF, Stewart JM, Boag DE, et al: The hypolipidemic action of probucol: a study of its effects on high and low density lipoproteins. J Lipid Res 24:588-595, 1983

28. Kesaniemi YA, Grundy SM: Influence of probucol on cholesterol and lipoprotein metabolism in man. J Lipid Res 25:780-790, 1984

29. Durrington PN, Miller JP: Double-blind, placebo-controlled, cross-over trial of probucol in heterozygous familial hypercholesterolaemia. Atherosclerosis 55:187-194, 1985

30. Baker SG, Joffe BI, Mendelsohn D, et al: Treatment of homozygous familial hypercholesterolemia with probucol. S Afr Med J 62:7-11, 1982

31. Naruszewicz M, Carew TE, Pittman RC, et al: A novel mechanism whereby probucol lowers low density lipoprotein levels demonstrated in the LDL receptor deficient rabbit. J Lipid Res 25:780-790, 1984

32. Marshall FN: Pharmacology and toxicology of probucol. Artery 10:7-21, 1982

33. Steinberg D: Studies on the mechanism of action of probucol. Am J Cardiol 57:16H-21H, 1986

34. Henriksen T, Mahoney EM, Steinberg D: Enhanced macrophage degradation of biologically modified low density lipoprotein. Arteriosclerosis 3:149-159, 1983

35. Parthasarathy S, Young SG, Witzum JL, et al: Probucol inhibits oxidative modification of low density lipoprotein. J Clin Invest 77:641-644, 1986

36. Mahley RW, Innerarity T, Rall SC, et al: Plasma lipoproteins: Apolipoprotein structure and function. J Lipid Res 25:1277. 1294,1984

37. Gregg R: Dysbetalipoproteinemia syndromes, in Proceedings of the Symposium on Lipoprotein Deficiency Syndromes. Adv Exp Med Biol 201:289-298, 1986

38. Bradley WA, Gianturco SH: Apo $E$ is necessary and sufficient for the binding of large triglyceride-rich lipoproteins to the LDL receptor; apo B is unnecessary. J Lipid Res 27:40-48, 1986 